\title{
WELL-POSEDNESS OF A RIEMANN-HILBERT PROBLEM ON $\boldsymbol{d}$-REGULAR QUASIDISKS
}

\author{
Eric Schippers and Wolfgang Staubach \\ University of Manitoba, Department of Mathematics \\ Winnipeg, Manitoba, R3T 2N2, Canada; eric.schippers@umanitoba.ca \\ Uppsala University, Department of Mathematics \\ Box 480, 75106 Uppsala, Sweden; wulf@math.uu.se
}

\begin{abstract}
We prove the well-posedness of a Riemann-Hilbert problem on $d$-regular quasidisks, with boundary data in a class of Besov spaces.
\end{abstract}

\section{Introduction}

Given a domain in the plane and a function defined on its boundary, a special case of the Riemann-Hilbert problem sometimes called the "jump problem" is to find holomorphic functions in the inside and the outside the domain whose difference of the boundary values is equal to that given function, on the boundary of the domain. To show the well-posedness of this problem amounts to proving the existence, the uniqueness and the continuous dependence on the data of the solution. As is wellknown, a quasidisk $\Omega$ is the image of the unit disk under a planar quasiconformal mapping. The boundary of $\Omega$, i.e. a quasicircle, is in general quite a rough fractal set which may not be rectifiable. This issue causes problems in dealing with boundary value problems in potential theory when the boundary data needs to belong to a suitable space defined on the rough boundary. For the case of the so called Weil-Petersson-class (or WP-class) quasicircles, Radnell, Schippers and Staubach demonstrated in [6] that the aforementioned quasicircles are rectifiable and that the corresponding quasidisk is a chord-arc domain. This enabled us to use Semmes' construction of the solution to the Riemann boundary value problem in [7], to show the well-posedness of the Riemann boundary value problem on WP-class quasicircles with initial data in a certain conformally invariant Besov space.

In this paper we establish the well-posedness of the aforementioned boundary value problem on what we call $d$-quasidisks, with boundary data in an appropriate class of Besov spaces. These are quasidisks which have the property that their boundaries are $d$-sets in the sense of Jonsson and Wallin $[3,4,11]$. The notion of Besov spaces, or the traces of Sobolev spaces, is well-defined on these sets. Although the Cauchy integral doesn't make sense directly on these sets, using Cauchy-Pompeiu's formula one can still get a solution to the boundary value problem with desired properties.

Notational convention. In this paper we shall denote all generic constants by $C$, even if the values of $C$ might change from line to line.

https://doi.org/10.5186/aasfm.2017.4210

2010 Mathematics Subject Classification: Primary 30C62, 30E25, 35Q15; Secondary 46E35.

Key words: Besov spaces, $d$-sets, quasidisks, Riemann boundary value problem.

Both authors are grateful for the financial support from the Wenner-Gren Foundations. Eric Schippers is also partially supported by the National Sciences and Engineering Research Council of Canada. 


\section{The analysis of the Riemann boundary value problem}

2.1. Sobolev and Besov spaces on $\boldsymbol{d}$-quasidisks. In what follows, we will use the following conventions for notation of domains. Let $\Gamma$ be a quasicircle in $\overline{\mathbf{C}}$. We will always assume $\infty \notin \Gamma$; we denote the bounded component of $\overline{\mathbf{C}} \backslash \Gamma$ by $\Omega^{+}$and the unbounded component by $\Omega^{-}$. In order to solve Riemann's boundary value problem, we need some machinery from geometric measure theory and theory of function spaces on rough domains which we shall briefly review below.

Let $\Omega$ be an open connected domain in the plane. For $1<p<\infty$, one denotes by $W^{1, p}(\Omega)$ the Sobolev space of complex valued functions in $L^{p}(\Omega)$ with

$$
\|h\|_{W^{1, p}(\Omega)}:=\left\{\|h\|_{L^{p}(\Omega)}^{p}+\left\|h^{\prime}\right\|_{L^{p}(\Omega)}^{p}+\left\|\bar{h}^{\prime}\right\|_{L^{p}(\Omega)}^{p}\right\}^{\frac{1}{p}}<\infty,
$$

where the derivations are in the sense of distributions. In case $\Omega$ is a domain that contains $\infty$, then we say $h \in W^{1, p}(\Omega)$ if $\left.h\right|_{\Omega \backslash\{\infty\}} \in W^{1, p}(\Omega \backslash\{\infty\})$, according to the above definition. One can also define the homogeneous Sobolev space $\dot{W}^{1, p}(\Omega)$ consisting of functions $h$ for which

$$
\|h\|_{\dot{W}^{1, p}(\Omega)}:=\left\{\left\|h^{\prime}\right\|_{L^{p}(\Omega)}^{p}+\left\|\bar{h}^{\prime}\right\|_{L^{p}(\Omega)}^{p}\right\}^{\frac{1}{p}}<\infty .
$$

Theorem 2.1. Let $\Omega^{+}$be a quasidisk and $\Omega^{-}$be its complement containing $\infty$. Then the following statements hold:

(1) If $h$ is a complex harmonic function in $\Omega^{+}$and $h \in \dot{W}^{1, p}\left(\Omega^{+}\right)$for $2 \leq p<\infty$, then $h \in W^{1, p}\left(\Omega^{+}\right)$.

(2) If $h$ is a complex harmonic function in $\Omega^{-}, h(\infty)=0$ and $h \in \dot{W}^{1, p}\left(\Omega^{-}\right)$for $2<p<\infty$, then $h \in W^{1, p}\left(\Omega^{-}\right)$.

Proof. Since $\Omega^{ \pm}$are simply connected, any complex harmonic function can be written as a sum of a holomorphic and an anti-holomorphic function. Therefore it is enough to prove the claim for holomorphic functions.

It is well-known, using a combination of the results in [8] and [9], that for any finitely connected Hölder domain $\Omega$ with finite area and any $z_{0}$ in $\Omega$, one has the analytic Poincaré inequality

$$
\left\|F-F\left(z_{0}\right)\right\|_{L^{p}(\Omega)} \leq C_{z_{0}}\left\|F^{\prime}\right\|_{L^{p}(\Omega)},
$$

for $2 \leq p<\infty$ and for any $F$ holomorphic in $\Omega$. Since $\Omega^{+}$and $\Omega^{-} \cap B(0, R)$ (for $R$ fixed but sufficiently large) are both finite-area, finitely connected Hölder domains (these are in fact both John domains, which are known to be Hölder domains), estimate (2.3) is valid for both of these domains.

To prove (1), it is enough to show that $h \in L^{p}\left(\Omega^{+}\right)$. Choose a point $z_{0} \in \Omega^{+}$. The Poincaré inequality above yields that for $2 \leq p<\infty$ and $h$ holomorphic in $\Omega^{+}$ and belonging to $\dot{W}^{1, p}\left(\Omega^{+}\right)$one has

$$
\begin{aligned}
\|h\|_{L^{p}\left(\Omega^{+}\right)} & \leq C\left\|h-h\left(z_{0}\right)\right\|_{L^{p}\left(\Omega^{+}\right)}+C|\Omega|^{\frac{1}{p}}\left|h\left(z_{0}\right)\right| \\
& \leq C\left(\left|h\left(z_{0}\right)\right|+\left\|h^{\prime}\right\|_{L^{p}\left(\Omega^{+}\right)}\right)<\infty
\end{aligned}
$$

where $\left|\Omega^{+}\right|$denotes the area of $\Omega^{+}$.

To prove (2) we observe that the holomorphicity of $h$ and its vanishing at $\infty$ imply that $h(z)=\frac{\psi(z)}{z}$ where $\psi$ is holomorphic and bounded on the set $|z|>R$, where $R$ is such that $\Omega^{+} \subset B(0, R)$. Therefore, for $p>2$, Poincaré's inequality for 
$\Omega^{-} \cap B(0, R)$ yields that

$$
\begin{aligned}
\|h\|_{L^{p}\left(\Omega^{-}\right)} \leq & \|h\|_{L^{p}\left(\Omega^{-} \cap B(0, R)\right)}+\|h\|_{L^{p}(\mathbf{C} \backslash B(0, R))} \\
\leq & C\left\|h-h\left(z_{0}\right)\right\|_{L^{p}\left(\Omega^{-} \cap B(0, R)\right)}+C\left|\Omega^{-} \cap B(0, R)\right|^{\frac{1}{p}}\left|h\left(z_{0}\right)\right| \\
& \left.+C \iint_{|z|>R}|z|^{-p} d A(z)\right) \\
\leq & C\left(\left|h\left(z_{0}\right)\right|+\left\|h^{\prime}\right\|_{L^{p}\left(\Omega^{-} \cap B(0, R)\right)}+1\right),
\end{aligned}
$$

for $h$ holomorphic in $\Omega^{-}$and any $z_{0} \in \Omega^{-} \cap B(0, R)$. This completes the proof of the theorem.

Corollary 2.2. If $u \in \dot{W}^{1, p}\left(\Omega^{-}\right)$is complex harmonic and $p \in(2, \infty)$, then $u \in W^{1, p}\left(\Omega^{-}\right)$iff $u(\infty)=0$.

Proof. If $u(\infty)=0$ then the result follows directly from part (2) of Theorem 2.1. On the other hand, if $u(\infty) \neq 0$ then there exist $r$ and $\varepsilon$ both greater than zero such that $|u(z)|>\varepsilon$ for $|z|>r$. This would prevent $u$ from being in $L^{p}\left(\left(\Omega^{-}\right)\right.$and therefore also from belonging to $W^{1, p}\left(\Omega^{-}\right)$.

In order to tackle the problem of boundary values on quasidisks which are not rectifiable, we need to restrict the class of quasidisks to those that are also so called $d$-sets.

Definition 2.3. Let $\Gamma$ be a Borel set in $\mathbf{R}^{2}$ and $0<d \leq 2$. We say that $\Gamma$ is a $d$-regular set (or a $d$-set for short) if it is bounded and if there is a constant $C_{\Gamma}$ such that

$$
\frac{1}{C_{\Gamma}} r^{d} \leq \mathscr{H}^{d}(B(x, r) \cap \Gamma) \leq C_{\Gamma} r^{d}
$$

for all $x \in \Gamma, 0<r \leq 1$, where $\mathscr{H}^{d}$ denotes the $d$-dimensional Hausdorff measure.

The 1-regular sets are usually referred to as Ahlfors-regular sets. From this, it also follows that the Hausdorff dimension $d_{\mathrm{H}}$ of an $d$-regular set is equal to $d$. For general quasicircles one has the following basic result due to Gehring and Väisälä [1].

Theorem 2.4. If $\Omega$ is a quasidisk in $\mathbf{R}^{2}$ then $\partial \Omega$ has Hausdorff dimension $d$ for some $1 \leq d<2$.

The following definition pinpoints the class of quasidisks that are considered in this paper.

Definition 2.5. We call a quasidisk $\Omega$ a $d$-quasidisk if its boundary $\partial \Omega$ is a $d$-regular set.

It follows from Theorem 2.4 that for a $d$-quasidisk one has that $d \in[1,2)$. This fact will be important for the existence of traces and extensions as we shall see below.

Below we will review some basic facts concerning Besov spaces on $d$-regular sets.

Definition 2.6. Let $\Gamma \subset \mathbf{R}^{2}$ be an $d$-regular set, $0<d \leq 2,1<p<\infty$, and $s \in(0,1)$. The Besov space $B_{p, p}^{s}(\Gamma)$ consists of all $u \in L^{p}(\Gamma)$ for which

$$
\iint_{|x-y|<1} \frac{|u(x)-u(y)|^{p}}{|x-y|^{d+p s}} d \mathscr{H}^{d}(x) d \mathscr{H}^{d}(y)<\infty
$$


The norm of this Besov space is defined by

$$
\|u\|_{B_{p, p}^{s}(\Gamma)}=\left\{\int_{\Gamma}|u(x)|^{p} d \mathscr{H}^{d}(x)+\iint_{|x-y|<1} \frac{|u(x)-u(y)|^{p}}{|x-y|^{d+p s}} d \mathscr{H}^{d}(x) d \mathscr{H}^{d}(y)\right\}^{1 / p} .
$$

In the study of the boundary value problems on quasicircles with initial data in Besov spaces which will be discussed in the next section, one encounters the so called $(\varepsilon, \delta)$-domains which were introduced by Jones [2]. We will not need the exact definition of $(\varepsilon, \delta)$-domains as they are somewhat technical, see [2]. But as was shown by Jones in [2], quasidisks and their complements are examples of $(\varepsilon, \delta)$-domains in $\mathbf{R}^{2}$. Jonsson [3, 4], and Jonsson and Wallin [5] showed that if $\Omega$ is an $(\varepsilon, \delta)$ domain in $\mathbf{R}^{2}$, whose boundary $\partial \Omega$ is an $d$-regular set, then the elements of $W^{1, p}(\Omega)$ have a well-defined trace or restriction to $\partial \Omega$. Furthermore, they showed various trace and extension properties for Sobolev and Besov space functions on $(\varepsilon, \delta)$-domains whose boundary is a $d$-set. Here we only need the following special case of the results in [11], originally proven for $(\varepsilon, \delta)$-domains with $d$-regular boundary.

Theorem 2.7. Let $\Gamma$ be the common boundary of a $d$-quasidisk $\Omega^{+}$and its complement $\Omega^{-}$. Then any function $f \in B_{p, p}^{1-\frac{2-d}{p}}(\Gamma)$ can be extended to a function $F \in W^{1, p}(\mathbf{C})$ in such a way that the limit

$$
\tilde{F}(z):=\lim _{r \rightarrow 0} \frac{1}{\pi r^{2}} \iint_{B(z, r)} F(\zeta) d A(\zeta)
$$

exists and $\tilde{F}(z)=f(z)$ for almost every $z \in \Gamma$. Furthermore, $\left.F\right|_{\Omega^{ \pm}} \in W^{1, p}\left(\Omega^{ \pm}\right)$and if $\Omega^{ \pm}(z, r):=\Omega^{ \pm} \cap B(z, r)$ and

$$
F_{ \pm}(z):=\lim _{r \rightarrow 0} \frac{1}{\left|\Omega^{ \pm}(z, r)\right|} \iint_{\Omega^{ \pm}(z, r)} F(\zeta) d A(\zeta)
$$

are the boundary values of the restrictions of $F$ to $\Omega^{ \pm}$, then $F_{ \pm}(z)=\tilde{F}(z)=f(z)$ (once again for almost all $z \in \Gamma$ ). Moreover, $\left\|\left.F\right|_{\Omega^{ \pm}}\right\|_{W^{1, p}\left(\Omega^{ \pm}\right)} \leq C\|f\|_{B_{p, p}^{1-\frac{2-d}{p}}{ }_{(\Gamma)}}$.

Thus the limit defining the boundary trace does not depend whether the approaching region is $\Omega^{+}$or its complement $\Omega^{-}$.

2.2. Well-posedness of the jump problem on $\boldsymbol{d}$-quasidisks. In this subsection, we show that the jump problem is solvable on $d$-quasidisks with boundary values in $B_{p, p}^{1-\frac{2-d}{p}}(\Gamma)$, with $p>2$, where $d$ is the regularity of the quasidisk. The solution is unique and depends continuously on the boundary data. The boundary of a $d$-quasidisk is in general not rectifiable, so Cauchy integrals will not make sense on general $d$-quasicircles. The wellposedness of the Riemann boundary value problem on rectifiable 1-quasidisks has been carried out in [6].

Thus we need to find a suitable substitute for the Cauchy integral. Given a $d$-quasidisk $\Omega^{+}$bounded by a quasicircle $\Gamma$ and a function $f \in B_{p, p}^{1-\frac{2-d}{p}}(\Gamma)$ with an extension $F$ in $W^{1, p}(\mathbf{C})$ (which exists by Theorem 2.7), using Cauchy-Pompeiu's formula one defines the Cauchy operator $J(\Gamma)$ by

$$
J(\Gamma) f(z):=F(z) \chi_{\Omega^{+}}(z)+\frac{1}{\pi} \iint_{\Omega^{+}} \frac{\bar{\partial} F(\zeta)}{\zeta-z} d A(\zeta), \quad z \in \mathbf{C} \backslash \Gamma,
$$

where $\chi_{\Omega^{+}}$denotes the characteristic function of the closure of $\Omega^{+}$. We will see that this definition is independent of the choice of the extension $F$, by the estimate 
(2.12) below, and the proof of the uniqueness part of Theorem 2.8. We will also need estimates for a certain integral operator that appears frequently in function theory. Given a domain $\Omega$ in the plane, this operator is defined by

$$
T_{\Omega} \varphi(z)=\iint_{\Omega} \frac{\varphi(\zeta)}{\zeta-z} d A(\zeta)
$$

The fact of particular interest to us in this context is that for a pair of complementary domains $\Omega^{+}$and $\Omega^{-}$, in the plane, one has for $1<p<\infty$ that

$$
\left\|T_{\Omega^{+}} \varphi\right\|_{\dot{W}^{1, p}\left(\Omega^{ \pm}\right)} \leq C\|\varphi\|_{L^{p}\left(\Omega^{+}\right)} .
$$

This is a direct consequence of the facts that $\partial\left(T_{\Omega^{+}} \varphi\right)(z)=\mathrm{P} \cdot \mathrm{V} \cdot \iint_{\Omega^{+}} \frac{\varphi(\zeta)}{(\zeta-z)^{2}} d A(\zeta)$, which is the Beurling transform of $\varphi \chi_{\Omega^{+}}$, and $\bar{\partial}\left(T_{\Omega^{+}} \varphi\right)(z)=-\pi \varphi(z) \chi_{\Omega^{+}}(z)$. Both derivatives here are in the sense of distributions. Now since the Beurling transform $B \varphi(z):=\mathrm{P} . \mathrm{V} \cdot \iint_{\mathbf{C}} \frac{\varphi(\zeta)}{(\zeta-z)^{2}} d A(\zeta)$ is a Calderón-Zygmund singular integral operator, it is well-known that $B$ is bounded on $L^{p}(\mathbf{C})$ for $1<p<\infty$. The estimate $(2.12)$ follows easily from these facts and the definition of the norm in (2.2).

Now we have all the ingredients to state and solve the following Riemann boundary value problem:

Theorem 2.8. Let $\Omega^{+}$be a $d$-quasidisk as above and let $f \in B_{p, p}^{1-\frac{2-d}{p}}(\Gamma)$, with $p>2$. Let $\Omega^{-}$denote the complement of the closure of $\Omega^{+}$in the Riemann sphere. Then there exist functions $u_{ \pm} \in \dot{W}^{1, p}\left(\Omega^{ \pm}\right)$with boundary values equal to $f_{ \pm}$on $\Omega^{ \pm}$, such that $f_{+}(w)-f_{-}(w)=f(w)$ for almost all $w \in \Gamma$. Moreover the solutions $u_{ \pm}$depend continuously on $f$. Finally, given two different solutions $u_{ \pm}$and $v_{ \pm}$of $f \in B_{p, p}^{1-\frac{2-d}{p}}(\Gamma)$, the difference $u_{+}-v_{+}=u_{-}-v_{-}$is a constant.

Proof. The proof will be divided in the following four steps:

1. Existence of the solution. We use Theorem 2.7 to extend $f$ to a function in $W^{1, p}(\mathbf{C})$ and then using $(2.10)$, we claim that the solution to the boundary value problem is given by

$$
u_{ \pm}=\left.(J(\Gamma) f)\right|_{\Omega^{ \pm}} .
$$

Let us first show that $J(\Gamma) f(z)$ is holomorphic in $\Omega^{+}$and $\Omega^{-}$. To see this, we observe that $\iint_{\Omega^{+}}|\bar{\partial} F(\zeta)| d A(\zeta)<\infty$, which follows from the fact that for $1<p<\infty$ and $\frac{1}{p}+\frac{1}{p^{\prime}}=1$

$$
\begin{aligned}
\iint_{\Omega^{+}}|\bar{\partial} F(\zeta)| d A(\zeta) & \leq\left|\Omega^{+}\right|^{\frac{1}{p^{\prime}}}\left(\iint_{\Omega^{+}}|\bar{\partial} F(\zeta)|^{p} d A(\zeta)\right)^{\frac{1}{p}} \\
& \leq C\|F\|_{W^{1, p}(\mathbf{C})} \leq C\|f\|_{B_{p, p}^{1-\frac{2-d}{p}}(\Gamma)}<\infty
\end{aligned}
$$

where the last step is a consequence of Theorem 2.7. Therefore, on $\Omega^{+}, \bar{\partial} J(\Gamma) f(z)=$ $\bar{\partial} F-\bar{\partial} F=0$, and on $\Omega^{-}, J(\Gamma) f(z)=\frac{1}{\pi} \iint_{\Omega^{+}} \frac{\bar{\partial} F(\zeta)}{\zeta-z} d A(\zeta)$ which is obviously holomorphic on $\Omega^{-} \backslash\{\infty\}$. From the expression (2.10) we also see that $J(\Gamma) f \rightarrow 0$ as $z \rightarrow \infty$, so in fact $J(\Gamma) f$ is holomorphic in a neighbourhood of $\infty$.

2. Continuous dependence on the initial datum. Estimates (2.12) for $\Omega^{ \pm}$and Theorem 2.7 yield

$$
\begin{aligned}
\left\|u_{+}\right\|_{\dot{W}^{1, p}\left(\Omega^{+}\right)} & =\left\|\left.(J(\Gamma) f)\right|_{\Omega^{+}}\right\|_{\dot{W}^{1, p}\left(\Omega^{+}\right)} \leq\|F\|_{\dot{W}^{1, p}\left(\Omega^{+}\right)}+C\|\bar{\partial} F\|_{L^{p}\left(\Omega^{+}\right)} \\
& \leq C\|F\|_{\dot{W}^{1, p}\left(\Omega^{+}\right)} \leq C\|f\|_{B_{p, p}^{1-\frac{2-d}{p}}(\Gamma)},
\end{aligned}
$$




$$
\begin{aligned}
\left\|u_{-}\right\|_{\dot{W}^{1, p}\left(\Omega^{-}\right)} & =\left\|\left.(J(\Gamma) f)\right|_{\Omega^{-}}\right\|_{\dot{W}^{1, p}\left(\Omega^{-}\right)} \leq C\|\bar{\partial} F\|_{L^{p}\left(\Omega^{+}\right)} \\
& \leq C\|F\|_{\dot{W}^{1, p}\left(\Omega^{+}\right)} \leq C\|f\|_{B_{p, p}^{1-\frac{2-d}{p}}} .(\Gamma)
\end{aligned}
$$

The estimates (2.14) and (2.15) show the continuous dependence of the solutions on the initial data $f$. Moreover by considering $u_{+}(z)-u_{-}(\infty)$ instead of $u_{+}$and $u_{-}(z)-u_{-}(\infty)$ instead of $u_{-}$and using Corollary 2.2, we can realize $u_{ \pm}$as elements in $W^{1, p}\left(\Omega^{ \pm}\right)$, with $p>2$.

3. Proof of the jump relation. Since by construction, the function $F$ in the definition of $J(\Gamma) f$ in formula (2.10) has the property that $\bar{\partial} F \in L^{p}(\mathbf{C})$, then the assumption that $p>2$ and a theorem due to Vekua [10, Theorem 1.19, p. 38], yield that the function $\iint_{\Omega^{+}} \frac{\bar{\partial} F(\zeta)}{\zeta-z} d A(\zeta)$ is continuous. Now regarding $u_{ \pm}$as a function in $W^{1, p}\left(\Omega^{ \pm}\right)$, and using Theorem 2.7 and the notations in the statement of Theorem 2.8, it is readily seen that the boundary value $f_{+}(w)$ of $u_{+}$is given by $f_{+}(w)=f(w)+$ $\frac{1}{\pi} \iint_{\Omega^{+}} \frac{\bar{\partial} F(\zeta)}{\zeta-w} d A(\zeta)$, with $w \in \Gamma$. The same reasoning also yields that the boundary value $f_{-}(w)$ of $u_{-}$is given by $f_{-}(w)=\frac{1}{\pi} \iint_{\Omega^{+}} \frac{\bar{\partial} F(\zeta)}{\zeta-w} d A(\zeta), w \in \Gamma$. Therefore, using the continuity result of Vekua mentioned above, one obtains the jump relation $f_{+}(w)-$ $f_{-}(w)=f(w)$ for $w \in \Gamma$.

4. Uniqueness of the solution. Once again, regarding $u_{ \pm}$and $v_{ \pm}$as elements in $W^{1, p}\left(\Omega^{ \pm}\right)$, we observe that the difference between the two jump decompositions with the same initial data is a function $\phi(z)$ which is holomorphic in $\overline{\mathbf{C}} \backslash \Gamma$. Moreover, $\phi$ 's restrictions to $\Omega^{ \pm}$belong to $\dot{W}^{1, p}\left(\Omega^{ \pm}\right)$, and the boundary values given by Theorem 2.7 are related by $\phi_{+}(z)=\phi_{-}(z)$. Moreover, according to Theorem 2.7 there exists a function $\psi \in W^{1, p}(\mathbf{C})$ which coincides with $\phi(z)$ in $\Omega^{-}$and $\psi^{\prime}$ 's limiting average according to (2.8) coincides with $\phi_{ \pm}$(defined using (2.9)) almost everywhere on $\Gamma$. The difference $\phi(z)-\psi(z):=\lambda(z)$ vanishes in $\Omega^{-}$, and its restriction to $\Omega^{+}$ belongs to the space $W^{1, p}\left(\Omega^{+}\right)$and $\lambda_{+}(w)=0$ for $w \in \Gamma$. Therefore, the proof of Theorem 3 in [11] yields that $\left.\lambda\right|_{\Omega^{+}}$is the limit in $W^{1, p}\left(\Omega^{+}\right)$of a sequence of functions $\lambda_{n} \in C_{0}^{\infty}\left(\Omega^{+}\right)$. Let us extend each function $\lambda_{n}$ to the entire plane by setting it equal to zero outside $\Omega^{+}$. Then the limit (in $W^{1, p}(\mathbf{C})$ ) of the resulting sequence of extended functions coincides with $\lambda$. Therefore $\lambda \in W^{1, p}(\mathbf{C})$ and so $\phi=\psi+\lambda \in W^{1, p}(\mathbf{C})$, and $\bar{\partial} \phi \in L^{p}(\mathbf{C})$ and vanishes outside $\Gamma$. Now since the 2 -dimensional Lebesgue measure of $\Gamma$ is zero, we have that $\bar{\partial} \phi=0$ in the sense of distributions. Using Weyl's lemma, i.e. that holomorphic distributions are holomorphic functions, one deduces that $\phi$ is a holomorphic function in $\mathbf{C}$. Since $\phi$ is the difference of two functions which are holomorphic at $\infty$, so is $\phi$. Thus $\phi$ is constant.

Remark 2.9. In fact $J(\Gamma) h$ is the unique solution which goes to zero at $\infty$.

Remark 2.10. At present the validity of Theorem 2.8 for $p=2$ is not known to us. There are of course two obstructions for this in the proof given in this paper, as is seen in the statement of Corollary 2.2 and in applying Vekua's theorem regarding continuity of the function $\iint_{\Omega^{+}} \frac{\bar{\partial} F(\zeta)}{\zeta-z} d A(\zeta)$. 


\section{References}

[1] Gehring, F., and J. VÄIsÄLÄ: Hausdorff dimension and quasiconformal mappings. - J. London Math. Soc. (2) 6, 1973, 504-512.

[2] Jones, P. W.: Quasiconformal mappings and extendability of functions in Sobolev spaces. Acta Math. 147:1-2, 1981, 71-88.

[3] Jonsson, A.: The trace of potentials on general sets. - Ark. Mat. 17:1, 1979, 1-18.

[4] Jonsson, A.: Besov spaces on closed subsets of $\mathbf{R}^{n}$. - Trans. Amer. Math. Soc. 341:1, 1994, 355-370.

[5] Jonsson, A., and H. Wallin: Function spaces on subsets of $\mathbf{R}^{n}$. - Math. Rep. 2:1, 1984.

[6] Radnell, D., E. Schippers, and W. Staubach: Dirichlet's problem and Sokhotski-Plemelj's jump formula on Weil-Petersson-class quasidisks. - Ann. Acad. Sci. Fenn. Math. 41:1, 2016, 119-127.

[7] Semmes, S. W.: Estimates for $(\bar{\partial}-\mu \partial)^{-1}$ and Calderón's theorem on the Cauchy integral. Trans. Amer. Math. Soc. 306:1, 1988, 191-232.

[8] Smith, W., and D. Stegenga: Hölder domains and Poincaré domains. - Trans. Amer. Math. Soc. 319:1, 1990, 67-100.

[9] Stanoyevitch, A., and D. Stegenga: Equivalence of analytic and Sobolev Poincaré inequalities for planar domains. - Pacific J. Math. 178:2, 1997, 363-375.

[10] Vekua, I. N.: Generalized analytic functions. - Pergamon Press, London-Paris-Frankfurt; Addison-Wesley Publishing Co., Inc., Reading, Mass., 1962.

[11] Wallin, W.: The trace to the boundary of Sobolev space on a snowflake. - Manuscripta Math. $73,1991,117-125$.

Received 7 April 2016 • Accepted 30 May 2016 